\title{
Editorial
}

\section{Translational Pathology of Early Cancer}

\author{
Sudhir Srivastava and William E. Grizzle \\ E-mail: srivasts@mail.nih.gov;wgrizzle@uab.edu
}

In the last decade, therapy for specific types of cancer has evolved from a standard treatment for each type of cancer to variable forms therapy, the choice of which is dependent upon the analysis and progression of the individual cancer. Characteristics of the tumor, from its clinical manifestation and stage to the cancer's molecular and pathologic features are beginning to play a major role in the selection of appropriate care of patients with malignant tumors. The visual pathologic features of tumors have always been the critical parameters in determining tumor type; however, over the last several decades molecular analysis of tumors has played an increasing role in the diagnostic pathology of tumors; thus, "molecular pathology" has gradually emerged as a discipline in pathology which exploits the use of nucleic acid based techniques such as DNA sequencing, fluorescent in-situ hybridization, real time quantitative polymerase chain reaction, nucleic acid microarrays for specialized studies, proteomic based techniques and antibody-based profiling of tissues and cells. Molecular pathology shares some aspects of practice with both anatomic and clinical pathology, and is sometimes considered a "crossover" discipline. Applications are found in studies of both hereditary and acquired disorders including inheritable genetic diseases, malignant tumors, hematological diseases (leukemias and lymphomas), and infectious diseases. For example, DNA testing is the most common method for identity testing with specific applications such as monitoring bone marrow engraftment, paternity testing, and forensic identification.

The transformation of normal cells to malignant cells involves multiple genetic and epigenetic changes that are currently being studied and defined. Many of these changes have already been defined; however, as these studies proceed, we should take advantage of any identified molecular changes to aid in the early detection of cancer and the corresponding preinvasive neoplastic lesions.

Small cancers may be successfully treated and even cured, and treating preinvasive neoplastic lesions may prevent the subsequent development of invasive cancer. Molecular detection offers many advantages in the detection of small tumors or preinvasive neoplastic changes. First, molecular methods will permit the identification of very small tumors in their earliest stages of development. These tumors can be treated successfully and cost effectively. Current methods of early detection, such as mammography, endoscopic examination with fine needle aspiration or other techniques of imaging, only detect cancers that have grown to a certain, albeit small size. In many cases, however, aggressive subtypes of small tumors may have already invaded blood and lymphatic vessels or have spread to regional lymph nodes. Secondly, molecular methods will eventually detect field changes especially those that affect broad epithelial areas. Because cancer often arises in fields primed for neoplastic development, in the future, treatments will be directed at field changes rather than at individual lesions. The ultimate objective of early detection, therefore, is the diagnosis of tumor initiation and the use of preventive methods to reverse field effects.

A primary goal of using molecular methods should be the ability to separate those preinvasive neoplastic lesions that are destined to progress from those that will not, avoiding the over treatment of harmless lesions. In diagnostic pathology, some lesions are diagnosed as 'borderline', 'atypical' or dysplastic or of indeterminate malignant potential; such terms reflect 
the uncertainty about the biological potential of these lesions. Very often, such lesions form a histologic continuum with definitive cancers; yet with current histologic methods, it is not possible in many cases to predict which lesions are likely to progress. In contrast, molecular methods will determine the biologic potential of tumors and their corresponding preinvasive neoplastic lesions. This is of key importance because the aggressiveness of preinvasive neoplastic lesions and of small tumors ultimately may guide treatment and subsequent patient management.

In the future, early detection largely may depend on the availability of highly specific molecular probes capable of being used to identify thousands of DNA sequences simultaneously. These probes may be used to detect altered DNA or other tumor products shed into the feces (colorectal cancer), sputum (lung, laryngeal and pharyngeal cancers), urine (bladder, renal or prostate cancers), and into the blood or saliva. They also may be used to analyze fluid from cysts (breast, pancreatic or thyroid cancer) or aid in diagnosis by fine needle aspiration. These probes will distinguish between neoplastic development, tissue repair (or regeneration), and cellular changes secondary to inflammation. They may even pinpoint the extent to which cells have progressed on the way to malignant transformation.

In addition to early detection, molecular markers may be useful in oncology in multiple other ways. A correlation of the molecular features with risk factors, clinical progression and demographics (e.g., age, race, sex) have clearly been identified as important in current medical care. In addition, the relationship between biomarkers and environmental exposure, family history of cancer and dietary history may provide important information about the biology and etiology of cancer. Biomarkers are currently in limited use as important intermediate endpoints in clinical and prevention trials; their use will expand to identify individuals who are at high risk for cancer, to select specific therapy, and to serve as prognostic factors which will provide more accurate estimates of clinical outcome.

If the use of biomarkers results in an increase in the discovery of small early stage tumors or of preinvasive neoplastic lesions that precede invasive cancer, there will be a profound impact on cancer related mortality. However, before specific molecular methods are used widely, their diagnostic value must first be confirmed and their practical benefit established compared to conventional morphology on which most medical decisions are currently made. For example, if a molecular method identifies only that prostate tumors with Gleason scores of 8,9 , and 10 are very aggressive lesions, the use of such a molecular method adds little to the current medical information about the aggressiveness of such tumors.

The purpose of this book is to describe the principles and the application of selected existing methods of molecular pathology and its use in the detection of early cancer. It will describe the molecular changes that characterize preinvasive neoplastic lesions, molecular targets for early detection, validation of molecular targets, and areas of new diagnostic technology which show promise either for early detection of neoplasias or other medically important uses in cancer prevention and care. The book describes recent advances in proteomics, metabolomics, stem cells research, micro RNA and other post-translational molecular modifications; all these will serve the pressing needs in the development of the practice of personalized medicine.

The chapters of the book are divided into three sections which follow a sequence from basic mechanisms of tumor development, through the molecular and biological abnormalities found in common types of cancers, to the technologies which are most applicable to detecting these biologic alterations in precancerous lesions. Each chapter discusses the scientific underpinnings of the evolution and progression of tumors in order to enable clinicians and other professionals who manage cancer patients to better understand the disease at the molecular and genetic levels.

The first section of this book focuses on the definition of preinvasive neoplasia, concepts in the detection of early cancer and cancer susceptibility; it describes molecular tools, mechanism of malignant transformation and familial predisposition to the development of cancer. As we know, cancer is a disease resulting from accumulated genetic and epigenetic alterations. To explore fully the detection of genetic changes as a tool for earlier detection of cancer, it is essential to understand the molecular pathogenesis of cancer, that is, the natural history of tumor progression at the molecular level; this permits the biological behavior of an evolving lesion (for example, dysplasia) or cancerization field changes to be predicted with greater accuracy. Current observations indicate that cancers usually evolve through many complex cellular processes, pathways, and networks including the genetic determinants of progression, regression, dormancy, and invasion. A better understanding of these pathways and associated alternative circuits is critical if we are to apply successfully molecular-based technologies to the early detection of cancer. 
The second section reviews the organ-specific cellular, molecular and genetic alterations that occur in various cancers specific to an organ or system. Knowledge of these molecular alterations can be exploited in developing novel approaches for cancer detection, chemoprevention and therapies. Research in molecular genetics, cell biology, protein chemistry, and immunology has found that cells and the immune system undergo many changes during neoplastic progression. Early neoplastic changes include, for example, production of novel proteins, growth factors, cytokines, immune suppressors (e.g., exosomes), etc., as well as gene regulation by genetic alterations and methylation. Because these changes have been consistently associated with malignant transformation, some of the molecular features are now recognized as biomarkers for cancer. Such biomarkers whether present in serum, urine, saliva or other bodily fluids should serve as indicators of early cancer or as risk markers for impending cancer.

The third section deals with the development of technologies for the detection of early neoplastic lesions. The technologies used in early detection of neoplasia are rapidly evolving while existing technologies are undergoing progressive refinement in their sensitivity, specificity, and throughput. Improved analytical tools have permitted a more detailed examination of the molecular basis of carcinogenesis and have provided the ability to identify the molecular and cellular signatures of cancer. Development of technologies to identify and quantitate molecular and genetic markers that are selectively expressed in pre-invasive neoplastic lesions and/or are correlated with invasive tumors is, therefore, crucial to early detection. For example, detection of widespread microsatellite instability (MSI) demonstrated by expansion or deletion of repeat elements of DNA in exfoliated cells, may be particularly useful for the detection of bladder, lung, and other cancers which shed cells into body fluids that can be sampled conveniently.

Several DNA-based techniques have been used to search for mutations in human cancer. With the advent of polymerase chain reaction (PCR) based detection of DNA and RNA from rare neoplastic cells in body fluids, mutations have been detected in ras and p53 genes of neoplastic cells in the stool of patients with colorectal and pancreatic cancer, in p53 from the urine of patients with bladder cancer, and in both ras and p53 from the sputum of patients with lung cancer. Methods such as single-strand conformation polymorphism (SSCP), clamped denaturing gel electrophoresis (CDGE), heteroduplex analysis, direct DNA se- quencing and other PCR techniques have been used to describe many of the molecular features of neoplasia. RNA based technologies include serial analysis of gene expression (SAGE), and chip analysis based on cDNA hybridization. Some protein-based assays have been developed to reveal frame-shift, splice-site and non-sense mutations by employing a coupled in vitro transcription/translation system. Protein-based assays can most easily detect genetic changes that ultimately result in truncated proteins. Molecular cytogeneticsbased technologies analyze genetic changes that occur before and during the histologic development of a tumor. Such methods are becoming powerful detection tools because of the ease of using these methodologies. For example, fluorescence in situ hybridization (FISH) using chromosome-specific peri-centrometric probes has been successful for detecting genetic abnormalities in solid tumors. FISH may even be performed on thin-paraffin sections with preserved morphology. Comparative Genomic Hybridization (CGH) provides information that is similar to conventional cytogenetics, but requires only DNA extracted from abnormal cells and does not require obtaining metaphase tumor cells for analysis. Again, CGH offers a powerful technique providing rapidly information as to alterations in chromosomes. As the resolution of CGH has increased, it has been successfully applied to discover a large amount of new cytogenetic information. Similarly, computerized cytomorphometric analysis represents a form of 'automated cytology'; it uses high speed computers coupled to modern high performance image analyzers to measure up to 128 nuclear features such as nuclear density and textural and structured nuclear features and measurements related to nuclear size and shape. In some sense, morphometric analysis is an integration of the effects of multiple molecular events on nuclear morphology and hence, combines computerized cytomorphometric analysis, an integrated analysis of pathological and molecular features. New developments include cytomorphometric analysis of nuclear features in 3 dimensions.

Even though many of these assays are technically challenging, the development of high throughput technologies that could speed analysis as well as integrate multiple channels of useful information is desirable. Also of importance, is portability of assays so that they can be performed reliably and consistently a multiple sites and can be adapted to a typical clinical laboratory at a referral hospital. Recent developments of microchip-based arrayed technologies for DNA, RNA, and protein detection are not only timely, but are bound 
to define a whole new era of cancer detection and diagnosis in molecular oncology.

We are grateful to the outstanding contributors who have described in this book their areas of investigation and expertise in the hope to stimulate dialogue among clinicians, basic scientists and other health professionals. We believe that in the future the information contained in this book will develop a foundation for more vigorous applied research in cancer detection, prevention and therapies. It is hoped that this book also will serve as an excellent up-to-date reference to all those who are interested in undertaking research on cancer detection and cancer susceptibility and in critically evaluating the extraordinary opportunities provided over the past three decades by the most remarkable progress in our knowledge of cancer biology. This great advance in our knowledge of cancer has resulted in the evolving field of molecular pathology together with molecular technology as the first step in translational research.

Sudhir Srivastava, Ph.D., M.P.H. William E. Grizzle, M.D., Ph.D. 